\section{WEB WATCH}

\section{Sharing PRIDE}

- http://www.ebi.ac.uk/pride/

With the rapid progress in the field of proteomics and the accumulation of vast amounts of protein information, there is an increasing need for this information to be brought together in a way that is easy to access and share. In response to this need, the European Bioinformatics Institute in the UK (which is part of the European Molecular Biology Laboratory) and the Flanders Interuniversity Institute for Biotechnology, Ghent University, Belgium, developed the PRoteomics IDEntifications database.

PRIDE provides an opensource database of protein identifications and has been designed to allow researchers to store, share and compare their results. This free-to-use database aims to provide access to published, peerreviewed, standardized data on protein identification from many different sources. PRIDE is closely linked to the Human Proteomics Organization's Proteomics Standards Initiative (HUPO-PSI) and will allow users to transfer data using the standards that are currently being developed as part of PSI.

The availability of the data in the same format allows the use of powerful computational analyses - for example, by comparing the proteins from a particular tissue under different conditions. Ultimately, this database will allow researchers to investigate how proteins are altered in many diseases, paving the way for new diagnostic and predictive methods.

The developers hope that the proteomics community will adopt PRIDE as the method of choice for making proteomics data freely available to, and exploitable by, the proteomics community. Large data sets including HUPO's Plasma Proteome project and a human platelet proteome set are currently available in PRIDE. And many more are undoubtedly on their way. ..

Ekat Kritikou

\title{
Choreographing the mitotic ballet
}
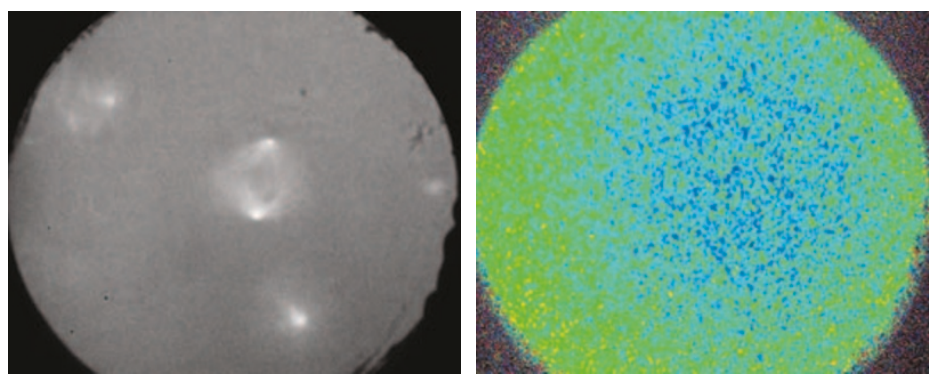

Spatial distribution of Alexa 488-Ran lifetime (top right) around a mitotic spindle (top left). Blue to green colouring reflects a decreasing interaction between RanGTP and importin- $\beta$. Image courtesy of Philippe Bastiaens, EMBL, Heidelberg, Germany.

During cell division, the chromosomal content is distributed to daughter cells by the mitotic spindle. Assembly of the mitotic spindle is tighly regulated and requires spatial cues. But what are the mechanisms that orchestrate this assembly? Using Xenopus laevis egg extracts - a system that is commonly used to study mitosis - Caudron et al. now show that spindle formation is coordinated by the generation of an intracellular

gradient of the small GTPase Ran at the chromosomes.

Two models have been put forward to explain how microtubules become organized in the mitotic spindle. The 'search and capture' model proposes that microtubules that grow from centrosomes are randomly captured and stabilized at the kinetochores on the chromosomes. However, an alternative model argues that chromatin changes the state of the mitotic cytoplasm in the surrounding area and promotes spindle assembly through a self-organization process. This model is based on the observation that Ran forms a chemical gradient from its GTP-bound active form (RanGTP), which is close to chromosomes, to a GDP-bound inactive form (RanGDP) in the cytoplasm. The high activity of the cytoplasmic Ran GTPase-activating protein (RanGAP) and a Ran guanine nucleotide-exchange factor (RanGEF or RCC1), which is localized on chromosomes, coordinate the two states of Ran. But is it possible that this gradient provides a positional signal that causes changes in microtubule dynamics and organizes the spindle around chromosomes?

To examine the formation of RanGTP-dependent gradients, the authors used a mathematical simulation to model the Ran gradient system. They showed that a long-range gradient of RanGTP that interacts with importin- $\beta$, and a short-range gradient of free RanGTP occur around chromatin. This long-range gradient occurs because the RanGTPimportin- $\beta$ complex prevents RanGAP-induced GTP hydrolysis

\section{PROTEIN DEGRADATION}

\section{Proteasome anchored}

The proteasomal machinery, which mediates ubiquitindependent proteolysis, degrades both cytoplasmic and nuclear protein targets, but how the proteasome accumulates in the nucleus has been elusive. Reporting in Cell, Takeda and Yanagida now provide mechanistic insight into how the proteasome localizes to the nucleus in fission yeast.

The Yanagida group had previously identified the nuclear envelope protein Cut8 as a promoting factor for the accumulation of nuclear proteasome. In the present study, the authors show that Cut8 has a short half-life and that the Lys residues in the $\mathrm{N}$ terminus of the protein become polyubiquitylated and are required for the rapid degradation of Cut8. They also demonstrate that Cut8 interacts with the proteasome, and that this interaction is enhanced by the ubiquitylated $\mathrm{N}$-terminal Lys residues.

So, could the interaction between the nuclear-envelopelocalized Cut 8 and the proteasome tether the proteasome to the nucleus? Indeed, this seems to be the case, as the proteasome accumulates in the nucleus in cells expressing wild-type Cut8, but not in cells expressing a Cut8 mutant that lacks the $\mathrm{N}$-terminal Lys residues.

Takeda and Yanagida identified the ubiquitin-conjugating enzyme Rhp6 and the ubiquitinligases Ubr1 and Rhp18 as the enzymes that are responsible for the polyubiquitylation and subsequent degradation of Cut8. Consistent with the observation that the ubiquitylation of Cut8 is essential for the nuclear enrichment of the proteasome, Rhp6- and Ubr1-null mutants showed much less nuclear accumulation of the proteasome. By contrast, in Rhp18-null mutants the accumulation of nuclear proteasome was only partly reduced. So, Cut8 is a target of Rhp6, Ubr1 and, maybe, Rhp18.

It remains unclear if the nuclear accumulation of the proteasome requires only the ubiquitylation of Cut 8 or whether it also requires its subsequent degradation, so this needs to be addressed in future research. The authors propose a feedback mechanism for the regulation of the level of Cut8 and 
RESEARCH H IGHLIGHTS

and diffuses away from the chromosomes. The authors argued that the long-range RanGTP-importin- $\beta$ gradient is physiologically relevant because it reflects the concentration gradient of released nuclear localization signal (NLS)-proteins that directly affect microtubule dynamics. Indeed, by analysing $X$. laevis egg extracts using fluorescence lifetime imaging microscopy (FLIM), they showed that RanGTP-dependent long-range gradients exist, such as the gradient of RanGTP-importin- $\beta$ and RanGTP-importin- $\beta$-RanBP1 (Ranbinding protein-1). Furthermore, microtubule nucleation and stabilization of the growing ends, which take place at different distances from the chromosomes, occur at significantly different concentrations of RanGTPimportin- $\beta$. Microtubule nucleation is restricted to a defined area around the chromosomes, whereas stabilization of the microtubule growing ends extends over longer distances.

The authors wanted to know whether the correct formation of a bipolar spindle around the chromosomes requires the RanGTP-importin- $\beta$ gradient. By experimentally varying the RanGTP-importin- $\beta$ gradient - using the addition of regulators of RanGTP production or RanGTP stability - they demonstrate that the correct gradient profile is important for proper spindle formation. A flattened gradient results in the loss of bipolar spindle assembly, the formation of random microtubule structures and a large decrease in microtubule-chromosome connections, indicating that the gradient has to operate within well-defined dimensions for the proper coordination of spindle assembly.

This elegant study illustrates how an intracellular chemical gradient can provide spatial information within a cell. The authors propose that the Ran system does not simply signal where the chromosomes are, but that it functions as a control element that spatially coordinates the self-organization of the microtubule-chromosome system. Whether intracellular gradients are also required for the organization of other structures within the cells remains to be investigated.

Ekat Kritikou

\section{(4) References and links} ORIGINAL RESEARCH PAPER Caudron M. et al. Spatial coordination of spindle assembly by chromosome-mediated signaling gradients Science 309, 1373-1376 (2005) WEB SITE

Eric Karsenti's web page: http://www-db.embl. de/jss/EmblGroupsOrg/g_40.html

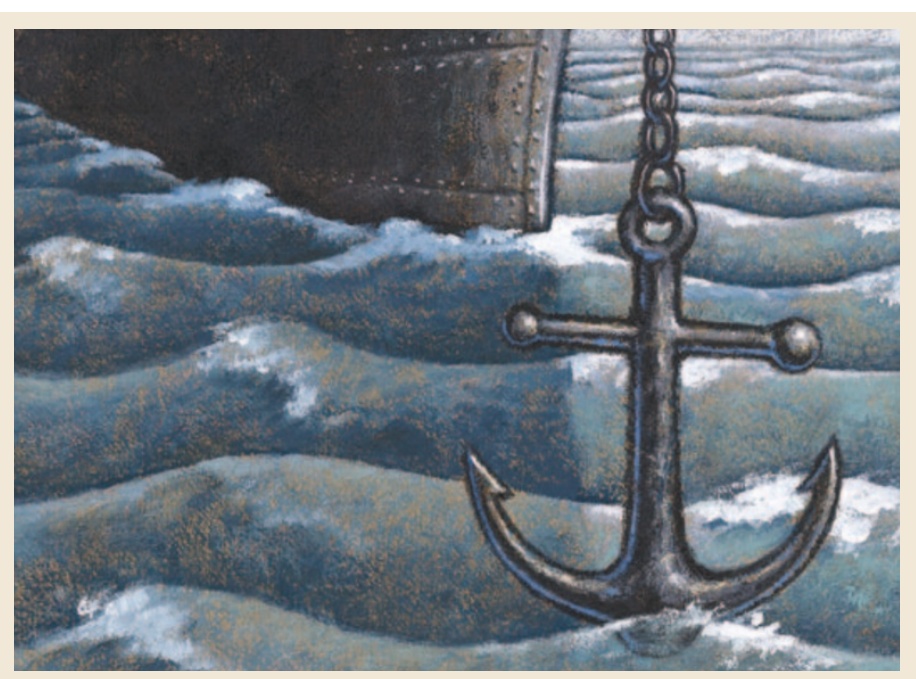

the nuclear proteasome, whereby Cut8 functions as a 'sensor' and 'anchor' for the proteasome - when Cut 8 becomes scarce, the amount of proteasome in the nucleus is reduced, which then feeds back to increase the amount of Cut8. This is followed by Cut8 ubiquitylation and subsequent tethering of nuclear proteasome.

Arianne Heinrichs

(2) References and links ORIGINAL RESEARCH PAPER Takeda, K. \& Yanagida, M. Regulation of nuclear proteasome by Rhp6/Ubc2 through ubiquitination and destruction of the sensor and anchor Cut8. Cell 122, 393-405 (2005)

\section{IN BRIEF}

\section{CHROMATIN REMODELLING}

\section{A 'loop recapture' mechanism for ACF-dependent nucleosome remodeling.}

Strohner, R. et al. Nature Struct. Mol. Biol. 12, 683-690 (2005)

\section{Chromatin remodeling through directional DNA translocation from an internal nucleosomal site.}

Saha, A. et al. Nature Struct. Mol. Biol. 12, 747-755 (2005)

Although many models for chromatin remodelling have been proposed, the mechanism remains elusive. Two recent papers, however, provide new insight into this essential process. Strohner et al. investigated the basis of nucleosome remodelling by the ISWI-containing ACF chromatin-remodelling complex, and propose a 'loop recapture' model in which DNA is lifted off the histone when ACF introduces a DNA loop into the nucleosome. In the second study, Saha et al. provide evidence that movement of DNA along the surface of the octamer occurs when the RSC/Sth1 remodelling complex binds the nucleosome core. On the basis of their findings, they propose a model whereby mobilization of the nucleosome involves directional DNA translocation, which leads to movement of the DNA relative to the histone octamer. Together, these studies suggest that remodelling enzymes function as specialized DNA translocases and track along the nucleosomal DNA while remaining anchored at the histone octamer.

\section{CELL CYCLE}

Multiple roles for separase auto-cleavage during the G2/M transition.

Papi et al. Nature Cell Biol. 4 Sep 2005 (doi:10.1038/ncb1303)

\section{Separase is required at multiple pre-anaphase cell cycle stages in human cells.}

Giménez-Abián et al. Cell Cycle 8 Sep 2005 (http://www.landesbioscience.com/ journals/cc/abstract.php?id=2147)

Cell division depends on the separation of sister chromatids in anaphase, while chromatid separation itself, at this stage in the cell cycle, is thought to depend on cleavage of chromosomebound cohesin by the protease separase. Evidence from yeast indicates that separase must also perform some function during interphase in mammalian cells. Two groups have studied the effects of separase cleavage in human cells at other cell-cycle stages - with interesting results. Papi et al. identified two major functions for the separase auto-cleavage reaction that occur at different stages in the cell cycle. First, they found that, during G2 phase, separase auto-cleavage promotes the destruction of the mitosis-inhibiting kinase WEE1, thereby allowing timely entry into M phase. Second, the cleaved forms of separase promote correct bipolar spindle assembly and proper sister-chromatid alignment during prometaphase. Giménez-Abián and colleagues also identified roles for separase at multiple cell-cycle stages, including interphase. Knockdown of separase revealed a delay or arrest at both G2 phase and prometaphase. In addition, they noted that the loss of chromatid cohesion as a result of separase activity might occur preferentially at the arms of the sister chromatids, but not at the centromeres. 